\title{
Two-Dimensional General Rate Model of Liquid Chromatography Incorporating Finite Rates of Adsorption-Desorption Kinetics and Core-Shell Particles
}

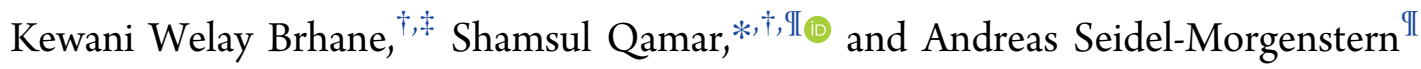 \\ ${ }^{\dagger}$ Department of Mathematics, COMSATS University Islamabad, Islamabad 45550, Pakistan \\ ${ }^{\ddagger}$ Department of Mathematics, Mekelle University, Mekelle, Ethiopia \\ ${ }^{\mathbb{I I}}$ Max Planck Institute for Dynamics of Complex Technical Systems, 39106 Magdeburg, Germany \\ Supporting Information
}

\begin{abstract}
A two-dimensional general rate model of liquid chromatography incorporating slow rates of adsorption-desorption kinetics, axial and radial dispersions, and core-shell particles is formulated. Radial concentration gradients are generated inside the column by considering different regions of injection at the inlet. Analytical solutions are obtained for a single-component linear model by simultaneously utilizing the Laplace and Hankel transformations for the considered two sets of boundary conditions. These linear solutions are useful for simulating liquid-chromatographic columns with diluted or small-volume samples and those in which radial concentration gradients are significant. To gain further insight into the process, analytical moments are also deduced from the Laplace-Hankel-domain

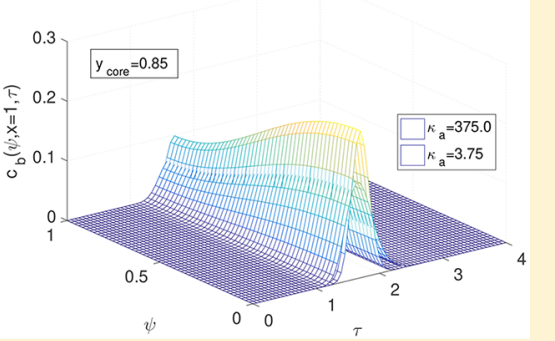
solutions. For situations of concentrated and large-volume samples, which are not solvable analytically, formulation of nonlinear models is necessary. In this study, a semidiscrete, high-resolution, finite-volume scheme is extended to approximate the resulting nonlinear-model equations for multicomponent mixtures. The performance of the column is analyzed by implementing a specified criterion of performance. A few numerical case studies are conducted to inspect the effects of the model parameters on the elution profiles.
\end{abstract}

\section{INTRODUCTION}

In liquid chromatography, the specific interactions of mixture components with the stationary phase play important roles in the separation. For that reason, various types of packing particles, such as fully porous particles, core-shell particles, nonporous inert particles, fully porous cylindrical fibers, nonporous cylindrical fibers, one-side-porous pellicular plates, nonporous plates, fully porous plates, two-side-porous pellicular plates, and hollow tubes, have been utilized as stationary phases for achieving faster separations and higher efficiencies of the columns. In analytical chromatography, nonporous packing particles provide faster separation than core-shell and fully porous particles. However, fully porous packing particles are widely used as compared with other packing particles, although they are subjected to intraparticle mass-transfer limitations, which reduce the efficiency of a column. Eventually, the use of core-shell particles as the stationary phase has been found advantageous over the use of nonporous and porous packing particles for achieving higher efficiencies and greater resolution of the components in a mixture. The thin porous layers on the solid impermeable cores provide shorter intraparticle-diffusion pathways, forcing the peaks of elution curves to be narrow. $^{2-7,9-16,19,20}$ Various theoretical studies exist in the literature for analytical chromatography using core-shell particles. ${ }^{1,2,6,8,13-18,20}$
In chromatography theory, mathematical modeling is an invaluable tool for studying dynamic processes inside a column without performing costly and time-consuming experiments. A significant number of models are available in the literature, allowing different levels of complexities. ${ }^{1,2,4,5,11,12}$ In the existing mathematical models, the general rate model (GRM) is an allinclusive model that incorporates many of the factors that influence the transfer of mass (e.g., external mass-transfer resistances, surface diffusion, kinetics of adsorption-desorption, axial dispersion, and pore diffusion). For some limits on kinetic parameters, the GRM reduces to a lumped kinetic model (LKM). ${ }^{7}$ A lumped kinetic model was used by Kaczmarski and Guiochon to study chromatographic columns packed with core-shell particles with thin porous layers by assuming the concentration inside the porous layers to be a single averaged value. ${ }^{15}$ Recently, our research group has derived analytical and numerical solutions of a quasistationary GRM for faster rates of adsorption and desorption kinetics. ${ }^{1,2}$

This article extends our previous theoretical investigations ${ }^{1,2}$ to a two-dimensional general rate model (2D-GRM) for slow rates of adsorption and desorption kinetics. Moreover, core-

Received: January 20, 2019

Revised: March 27, 2019

Accepted: April 22, 2019

Published: April 22, 2019 
shell particles with varying layer thicknesses are comprehensively included in the model equations. To trigger radial concentration gradients, different regions of injection are considered at the inlet of a column, such as inner- and outerannular-ring injections. Analytical solutions are obtained for a single-solute linear model through integrated application of the Laplace and Hankel transformations for the two considered types of inlet boundary conditions: the Danckwerts and Dirichlet boundary conditions. The analytically derived solutions are useful for simulating processes involving diluted or small volumes of injected samples. To gain further insight into the process, analytical moments are also extracted from the Laplace-Hankel domains of the solutions. For concentrated and large-volume samples, which are unsolvable analytically, formulation of nonlinear models is necessary. A semidiscrete, high-resolution, finite-volume scheme is extended for the approximation of the resulting nonlinear-model equations for multicomponent mixtures. The column performance is analyzed through a specific performance criterion. A few numerical tests are conducted to inspect the effects of various parameters on the elution profiles.

The major novelties of this article include (i) the formulation of 2D-GRM incorporating finite rates of adsorption-desorption kinetics and core-shell particles; (ii) the derivation of analytical solutions of the model for two sets of boundary conditions; (iii) the derivation of analytical expressions for temporal moments; (iv) the application of two-dimensional, high-resolution, finitevolume scheme to numerically solve the multicomponent, nonlinear 2D-GRM; and (v) the consideration of different case studies to quantify the effects of the rate constants for adsorption and desorption, the core-radius fraction, the axial and radial dispersions, the film mass-transfer resistance, the intraparticlediffusion resistance, the nonlinearity coefficients, and the inlet boundary conditions on the elution profiles.

The contents of this paper are organized in the following manner. The mathematical model of the problem (2D-GRM) is presented in Section 2. The analytical solutions of the linear model are obtained for a single-component mixture in Section 3. Section 4 is devoted to the derivation of the analytical moments. In Section 5, the numerical method is employed to find the approximate solutions of the nonlinear 2D-GRM. A criterion for the evaluation of process performance is explained in Section 6. Numerical test cases are given in Section 7. Finally, the conclusion of the study is presented in Section 8.

\section{MATHEMATICAL MODEL}

Consider an initially empty cylindrical column packed with core-shell particles. Assume that the radius of the column is $R$, and the radius of each packing particle is $R_{\mathrm{p}}$. Let the inert coreradius of each packing particle be $R_{\text {core }}$ and assume that the mixture contains $N_{\mathrm{c}}$ components. The model has three types of governing mass-balance equations.

The first equation expresses the concentration of the $i^{\text {th }}$ component of a mixture in the bulk phase. Thus, for $i=1,2$, ..., $N_{\mathcal{c}}$ it is given as

$$
\begin{aligned}
\frac{\partial c_{\mathrm{b}, i}}{\partial t}+u \frac{\partial c_{\mathrm{b}, i}}{\partial z}= & D_{z, i} \frac{\partial^{2} c_{\mathrm{b}, i}}{\partial z^{2}}+D_{\rho, i}\left(\frac{\partial^{2} c_{\mathrm{b}, i}}{\partial \rho^{2}}+\frac{1}{\rho} \frac{\partial c_{\mathrm{b}, i}}{\partial \rho}\right) \\
& -\frac{3 F \kappa_{\mathrm{ext}, i}}{R_{\mathrm{p}}}\left(c_{\mathrm{b}, i}-\left.c_{\mathrm{p}, i}\right|_{r=R_{\mathrm{p}}}\right)
\end{aligned}
$$

where $c_{\mathrm{b}, i}$ represents the concentration of the $i^{\text {th }}$ component in the bulk phase, $c_{\mathrm{p}, i}$ represents the concentration of the $i^{\text {th }}$ component inside the particles pores, $u$ denotes the interstitial velocity, $D_{z, i}$ is coefficient of the axial dispersion of the $i^{\text {th }}$ component, $F=\frac{1-\epsilon_{\mathrm{b}}}{\epsilon_{\mathrm{b}}}$ denotes the phase ratio, $\epsilon_{\mathrm{b}}$ is the external porosity, $D_{\rho, i}$ represents the radial-dispersion coefficient of the $i^{\text {th }}$ component, $\kappa_{\text {ext }, i}$ denotes the coefficient of external mass transfer of the $i^{\text {th }}$ component, $\rho$ is the column cylindrical coordinate, $r$ is the radial coordinate of the packing particles, and $z$ and $t$ are the column axial and time coordinates. ${ }^{1-3}$

The second equation describes a change in the concentration of $i^{\text {th }}$ component in the particle pores. Thus, for $i=1,2, \ldots, N_{\mathcal{c}}$, we obtain

$$
\begin{aligned}
& \epsilon_{\mathrm{p}} \frac{\partial c_{\mathrm{p}, i}}{\partial t}+\left(1-\epsilon_{\mathrm{p}}\right) \frac{\partial q_{\mathrm{p}, i}}{\partial t} \\
& \quad=\frac{1}{r^{2}} \frac{\partial}{\partial r}\left[r^{2}\left(\epsilon_{\mathrm{p}} D_{\mathrm{p}, i} \frac{\partial c_{\mathrm{p}, i}}{\partial r}+\left(1-\epsilon_{\mathrm{p}}\right) D_{\mathrm{s}, i} \frac{\partial q_{\mathrm{p}, i}}{\partial r}\right)\right]
\end{aligned}
$$

Here, $q_{\mathrm{p}, i}$ is the concentration of the $i^{\text {th }}$ component in the stationary phase, $\epsilon_{\mathrm{p}}$ is the internal porosity, $D_{\mathrm{p}, i}$ is the pore diffusivity of the $i^{\text {th }}$ component, and $D_{\mathrm{s}, i}$ is the surface diffusivity of the $i^{\text {th }}$ component. ${ }^{1-3}$

The third equation describes the concentration of the $i^{\text {th }}$ component adsorbed on the stationary phase and is given as

$$
\begin{aligned}
& \frac{\partial q_{\mathrm{p}, i}}{\partial t}=K_{\mathrm{a}, i}\left[c_{\mathrm{p}, i} q_{\mathrm{m}}-c_{\mathrm{p}, i} \sum_{j=1}^{N_{\mathrm{c}}} q_{\mathrm{p}, i}\right]-K_{\mathrm{d}, i} q_{\mathrm{p}, i} \\
& i=1,2, \ldots, N_{\mathrm{c}}
\end{aligned}
$$

In the equation given above, $K_{\mathrm{a}, i}$ is the adsorption rate constant for $i^{\text {th }}$ component, $K_{\mathrm{d}, i}$ the desorption rate constant of the $i^{\text {th }}$ component, and $q_{\mathrm{m}}$ is the saturation capacity of adsorption. We have assumed that all components have the same saturation capacity of adsorption.

To simplify the model equations and to minimize the number of the model parameters, a few dimensionless parameters are established as given below:

$$
\begin{array}{lll}
\tau=\frac{u t}{L} & x=\frac{z}{L} \quad y=\frac{r}{R_{\mathrm{p}}} \\
y_{\text {core }}=\frac{R_{\text {core }}}{R_{\mathrm{p}}} & \gamma=\frac{y-y_{\text {core }}}{1-y_{\text {core }}} & \psi=\frac{\rho}{R} \\
\kappa_{\mathrm{a}, i}=\frac{K_{\mathrm{a}, i} q_{\mathrm{m}} L}{u} & a_{i}=\frac{K_{\mathrm{a}, i} q_{\mathrm{m}}}{K_{\mathrm{d}, i}} \quad b_{i}=\frac{a_{i}}{q_{\mathrm{m}}} \\
P e_{z, i}=\frac{L u}{D_{z, i}} & P e_{\rho, i}=\frac{R^{2} u}{D_{\rho, i} L} \quad \eta_{\mathrm{p}, i}=\frac{D_{\mathrm{p}, i} L}{u R_{\mathrm{p}}^{2}} \\
\eta_{\mathrm{s}, i}=\frac{D_{\mathrm{s}, i} L}{u R_{\mathrm{p}}^{2}} & B i_{\mathrm{p}, i}=\frac{\kappa_{\mathrm{ext}, i} R_{\mathrm{p}}}{D_{\mathrm{p}, i}} & B i_{\mathrm{s}, i}=\frac{\kappa_{\mathrm{ext}, i} R_{\mathrm{p}}}{D_{\mathrm{s}, i}} \\
\xi_{i}=\frac{3 F \kappa_{\mathrm{ext}, i} L}{R_{\mathrm{p}} u} &
\end{array}
$$

In the equations given above, $b_{i}$ represents the extent of nonlinearity, $P e_{z, i}$ represents the axial Peclet number, $P e_{\rho, i}$ represents the radial Peclet number, and $B i_{\mathrm{s}, i}$ and $B i_{\mathrm{p}, i}$ are the 
Biot numbers corresponding to the $i^{\text {th }}$ component of a mixture. Moreover, $y_{\text {core }} \in[0,1]$ stands for the core-radius fraction. The particles are fully porous for $y_{\text {core }}=0$, whereas they are coreshell particles for $0<y_{\text {core }}<1$.

Using the dimensional quantities given in eq 4 , the governing eqs $1-3$ can be written as

$$
\begin{aligned}
& \frac{\partial c_{\mathrm{b}, i}}{\partial \tau}+\frac{\partial c_{\mathrm{b}, i}}{\partial x}=\frac{1}{P e_{z, i}} \frac{\partial^{2} c_{\mathrm{b}, i}}{\partial x^{2}}+\frac{1}{P e_{\rho, i}}\left[\frac{\partial^{2} c_{\mathrm{b}, i}}{\partial \psi^{2}}+\frac{1}{\psi} \frac{\partial c_{\mathrm{b}, i}}{\partial \psi}\right] \\
& -\xi_{i}\left(c_{\mathrm{b}, i}-\left.c_{\mathrm{p}, i}\right|_{\gamma=1}\right) \\
& \epsilon_{\mathrm{p}} \frac{\partial c_{\mathrm{p}, i}}{\partial \tau}+\left(1-\epsilon_{\mathrm{p}}\right) \frac{\partial q_{\mathrm{p}, i}}{\partial \tau} \\
& =\frac{2}{\gamma\left(1-y_{\text {core }}\right)^{2}+y_{\text {core }}\left(1-y_{\text {core }}\right)} \\
& \times\left[\epsilon_{\mathrm{p}} \eta_{\mathrm{p}, i} \frac{\partial c_{\mathrm{p}, i}}{\partial \gamma}+\left(1-\epsilon_{\mathrm{p}}\right) \eta_{\mathrm{s}, i} \frac{\partial q_{\mathrm{p}, i}}{\partial \gamma}\right] \\
& +\frac{1}{\left(1-y_{\text {core }}\right)^{2}}\left[\epsilon_{\mathrm{p}} \eta_{\mathrm{p}, i} \frac{\partial^{2} c_{\mathrm{p}, i}}{\partial \gamma^{2}}+\left(1-\epsilon_{\mathrm{p}}\right) \eta_{\mathrm{s}, i} \frac{\partial^{2} q_{\mathrm{p}, i}}{\partial \gamma^{2}}\right] \\
& \frac{\partial q_{\mathrm{p}, i}}{\partial \tau}=\frac{\kappa_{\mathrm{a}, i}}{a_{i}}\left[c_{\mathrm{p}, i}\left(a_{i}-b_{i} \sum_{j=1}^{N_{\mathrm{c}}} q_{\mathrm{p}, j}\right)-q_{\mathrm{p}, i}\right]
\end{aligned}
$$

For sufficiently higher values of $\kappa_{\mathrm{a}, i}$, the kinetic equation in eq 7 reduces to the following Langmuir isotherm describing the equilibrium concentration of the solute in the stationary phase: $:^{3,26}$

$$
q_{\mathrm{p}, i}=\frac{a_{i} c_{\mathrm{p}, i}}{1+\sum_{j=1}^{N_{\mathrm{c}}} b_{j} c_{\mathrm{p}, j}}
$$

The governing equations presented in eqs 5-7 are solved for the following initial and boundary conditions. For an initially empty column, the initial conditions (ICs) are given as

$$
\begin{aligned}
& c_{\mathrm{b}, i}(\tau=0, \psi, x)=0 \\
& c_{\mathrm{p}, i}(\tau=0, \gamma, \psi, x)=0=q_{\mathrm{p}, i}(\tau=0, \gamma, \psi, x) \\
& \forall x, \psi, \gamma \in(0,1)
\end{aligned}
$$

Along the dimensionless radial coordinate of the column at $\psi$ $=0$ and $\psi=1$, the following boundary conditions (BCs) can be used to solve eq 5:

$$
\begin{aligned}
& \left.\frac{\partial c_{\mathrm{b}, i}}{\partial \psi}\right|_{\psi=0}=0 \\
& \left.\frac{\partial c_{\mathrm{b}, i}}{\partial \psi}\right|_{\psi=1}=0
\end{aligned}
$$

Along the dimensionless radial coordinate of the packing particles at $\gamma=0$ and $\gamma=1$, the following BCs can be used to solve eq 6:

$$
\begin{aligned}
& \left.\frac{\partial c_{\mathrm{p}, i}}{\partial \gamma}\right|_{\gamma=0}=0 \\
& \frac{\epsilon_{\mathrm{p}}}{B i_{\mathrm{p}, i}} \frac{\partial c_{\mathrm{p}, i}}{\partial \gamma}+\left.\frac{\left(1-\epsilon_{\mathrm{p}}\right)}{B i_{\mathrm{s}, i}} \frac{\partial q_{\mathrm{p}, i}}{\partial \gamma}\right|_{\gamma=1} \\
& \quad=\left(1-y_{\text {core }}\right)\left(c_{\mathrm{b}, i}-c_{\mathrm{p}, i}{ }_{\gamma=1}\right)
\end{aligned}
$$

At the inlet and outlet of the column, the following types of BCs are applied on eq 1 .

Type I: Dirichlet Boundary Conditions. The boundary conditions for an injection through an inner annular region have the forms:

$$
\left.c_{\mathrm{b}, i}\right|_{x=0}=\left\{\begin{array}{llll}
c_{i}^{\mathrm{inj}}, & 0 \leq \psi \leq \widetilde{\psi} & \text { and } & 0 \leq \tau \leq \tau_{\mathrm{inj}} \\
0, & \widetilde{\psi}<\psi \leq 1 & \text { or } & \tau>\tau_{\mathrm{inj}}
\end{array}\right.
$$

The boundary conditions for an outer-annular-region injection have the expressions:

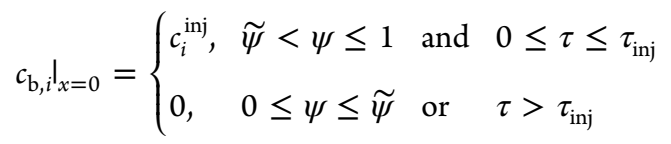

Assuming a column of hypothetically infinite length, the following Neumann boundary condition can be used at the column exit:

$$
\left.\frac{\partial c_{\mathrm{b}, i}}{\partial x}\right|_{x=\infty}=0
$$

The variable $\tau_{\text {inj }}$ denotes the injection time of the sample, and $c_{i}^{\text {inj }}$ denotes the concentration of the injected sample.

Type II: Danckwerts Boundary Conditions. The boundary conditions for an injection through the inner annular region are

$$
\begin{aligned}
& -\frac{1}{P e_{z, i}} \frac{\partial c_{\mathrm{b}, i}}{\partial x}+\left.c_{\mathrm{b}, i}\right|_{x=0} \\
& =\left\{\begin{array}{llll}
c_{i}^{\text {inj }}, & 0 \leq \psi \leq \widetilde{\psi} & \text { and } & 0 \leq \tau \leq \tau_{\text {inj }} \\
0, & \widetilde{\psi}<\psi \leq 1 \text { or } & \tau>\tau_{\text {inj }}
\end{array}\right.
\end{aligned}
$$

The boundary conditions for outer-annular-region injection are given by

$$
\begin{aligned}
& -\frac{1}{P e_{z, i}} \frac{\partial c_{\mathrm{b}, i}}{\partial x}+\left.c_{\mathrm{b}, i}\right|_{x=0} \\
& =\left\{\begin{array}{llll}
c_{i}^{\text {inj }}, & \widetilde{\psi}<\psi \leq 1 \text { and } & 0 \leq \tau \leq \tau_{\text {inj }} \\
0, & 0 \leq \psi \leq \widetilde{\psi} & \text { or } & \tau>\tau_{\text {inj }}
\end{array}\right.
\end{aligned}
$$

For a column of finite length, the considered Neumann boundary conditions are given by

$$
\left.\frac{\partial c_{\mathrm{b}, i}}{\partial x}\right|_{x=1}=0
$$

\section{ANALYTICAL SOLUTIONS OF THE PROBLEM}

In this section, the analytical solutions of a single-solute $\left(N_{c}=1\right)$ linear model are derived for the aforementioned boundary 
conditions. It is assumed that loading of a solute is significantly low for all times in such a way that the available number of adsorption sites does not vary as the solute passes through a column. Thus, the extent of the nonlinearity coefficient becomes negligible (i.e., $b=0$ ), or the adsorption-saturation capacity $\left(q_{\mathrm{m}}\right)$ is very large. With this assumption, eq 7 takes the following linear form:

$$
\frac{\partial q_{\mathrm{p}}}{\partial \tau}=\frac{\kappa_{\mathrm{a}}}{a}\left(a c_{\mathrm{p}}-q_{\mathrm{p}}\right)
$$

Let the zeroth-order Hankel transform of $c_{\mathrm{b}}$ and $c_{\mathrm{p}}$ with respect to $\psi$ be $c_{\mathrm{bH}}$ and $c_{\mathrm{pH}}$ with a Hankel parameter $k^{2}$. Then, the Hankel transform of eq 5 with respect to $\psi$ is given by

$$
\frac{\partial c_{\mathrm{bH}}}{\partial \tau}+\frac{\partial c_{\mathrm{bH}}}{\partial x}=\frac{1}{P e_{z}} \frac{\partial^{2} c_{\mathrm{bH}}}{\partial x^{2}}-\frac{k^{2} c_{\mathrm{bH}}}{P e_{\rho}}-\xi\left(c_{\mathrm{bH}}-c_{\mathrm{pH}} \mathrm{l}_{\gamma=1}\right)
$$

Again, let the Laplace transform of $c_{\mathrm{bH}}$ and $c_{\mathrm{pH}}$ with respect to $\tau$ be $\bar{c}_{\mathrm{bH}}$ and $\bar{c}_{\mathrm{pH}}$ with a Laplace parameter $s^{2}$. Then, the Laplace transform of eq 19 is found to be

$$
\begin{aligned}
& \frac{1}{P e_{z}} \frac{\mathrm{d}^{2} \bar{c}_{\mathrm{bH}}}{\mathrm{d} x^{2}}-\frac{\mathrm{d} \bar{c}_{\mathrm{bH}}}{\mathrm{d} x}-\left(s+\frac{k^{2}}{P e_{\rho}}\right) \bar{c}_{\mathrm{bH}}-\xi\left(\bar{c}_{\mathrm{bH}}-\bar{c}_{\mathrm{pH}} \mathrm{l}_{\gamma=1}\right) \\
& \quad=0
\end{aligned}
$$

In a similar manner, the application of Hankel and Laplace transforms on eqs 6 and 18 give, after some manipulations, the following simplified differential equation:

$$
\begin{aligned}
& \frac{\mathrm{d}^{2} \bar{c}_{\mathrm{pH}}}{\mathrm{d} \gamma^{2}}+\frac{2\left(1-y_{\text {core }}\right)^{2}}{\gamma\left(1-y_{\text {core }}\right)^{2}+y_{\text {core }}\left(1-y_{\text {core }}\right)} \frac{\mathrm{d} \bar{c}_{\mathrm{pH}}}{\mathrm{d} \gamma}-A(s) \bar{c}_{\mathrm{pH}} \\
& \quad=0
\end{aligned}
$$

where

$$
A(s)=s\left(1-y_{\text {core }}\right)^{2}\left\{\frac{\epsilon_{\mathrm{p}}+\left[\left(1-\epsilon_{\mathrm{p}}\right) \kappa_{\mathrm{a}}\right] /\left(s+\kappa_{\mathrm{a}} / a\right)}{\epsilon_{\mathrm{p}} \eta_{\mathrm{p}}+\left[\left(1-\epsilon_{\mathrm{p}}\right) \eta_{\mathrm{s}} \kappa_{\mathrm{a}}\right] /\left(s+\kappa_{\mathrm{a}} / a\right)}\right\}
$$

For arbitrary constants $C_{1}$ and $C_{2}$, the solution to eq 22 is given by

$$
\begin{aligned}
\bar{c}_{\mathrm{pH}}(s, \gamma, k, x)= & \frac{1}{\gamma\left(1-y_{\text {core }}\right)^{2}+y_{\text {core }}\left(1-y_{\text {core }}\right)} \\
& \times\left\{C_{1} \exp \left[-\left(1-y_{\text {core }}\right) \sqrt{A(s)} \gamma\right]\right. \\
& \left.+C_{2} \frac{\exp \left[\left(1-y_{\text {core }}\right) \sqrt{A(s)} \gamma\right]}{2\left(1-y_{\text {core }}\right) \sqrt{A(s)}}\right\}
\end{aligned}
$$

By applying the Hankel and Laplace transforms to the BC at $\gamma$ $=0$ in eq 11 and substituting it in eq 23, we obtain

$$
C_{1}=\frac{y_{\text {core }} \sqrt{A(s)}-1}{2 \sqrt{A(s)}\left(1-y_{\text {core }}\right)\left[y_{\text {core }} \sqrt{A(s)}+1\right]}
$$

On substituting eq 24 in eq 23 and then using the Hankel and Laplace transforms of $\mathrm{BC}$ at $\gamma=1$ in eq 11 , the resulting expression for $C_{2}$ comes out to be

$$
\begin{aligned}
C_{2}= & \left\{2 B(s) \sqrt{A(s)}\left[1-y_{\text {core }}\right]^{2}\left[1+y_{\text {core }} \sqrt{A(s)}\right]\right. \\
& {\left.\left[\bar{c}_{\mathrm{bH}}-\left.\bar{c}_{\mathrm{pH}}\right|_{\gamma=1}\right]\right\} } \\
& /\left\{1-y_{\text {core }}+\sqrt{A(s)}\left[1-y_{\text {core }} \sqrt{A(s)}\right]\right. \\
& +\left[1+y_{\text {core }} \sqrt{A(s)}\right][\sqrt{A(s)}-1] \\
& \left.\exp \left[2\left(1-y_{\text {core }}\right) \sqrt{A(s)}\right]\right\}
\end{aligned}
$$

where

$$
B(s)=\frac{B i_{\mathrm{p}} B i_{\mathrm{s}}}{\epsilon_{\mathrm{p}} B i_{\mathrm{s}}+\left(1-\epsilon_{\mathrm{p}}\right) B i_{\mathrm{p}}\left[\kappa_{\mathrm{a}} /\left(s+\kappa_{\mathrm{a}} / a\right)\right]}
$$

Evaluation of the solution in eq 23 at $\gamma=1$ leads us to the expression

$$
\bar{c}_{\mathrm{pH}} \mathrm{l}_{\gamma=1}=D(s)\left(\bar{c}_{\mathrm{bH}}-\bar{c}_{\mathrm{pH}} \mathrm{l}_{\gamma=1}\right)
$$

where

$$
\begin{aligned}
D(s)= & \left\{B ( s ) \left[y_{\text {core }} \sqrt{A(s)}-1+\left[y_{\text {core }} \sqrt{A(s)}+1\right]\right.\right. \\
& \left.\exp \left[2\left(1-y_{\text {core }}\right) \sqrt{A(s)}\right]\right\} \\
& /\left\{\left[1-y_{\text {core }} \sqrt{A(s)}\right][\sqrt{A(s)}+1]\right. \\
& +\left[1+y_{\text {core }} \sqrt{A(s)}\right][\sqrt{A(s)}-1] \\
& \left.\exp \left[2\left(1-y_{\text {core }}\right) \sqrt{A(s)}\right]\right\}
\end{aligned}
$$

Then, by applying Hankel transform on eq 27 and substituting into eq 20, we have the following second-order ordinary differential equation:

$$
\frac{\mathrm{d}^{2} \bar{c}_{\mathrm{bH}}}{\mathrm{d} x^{2}}-P e_{z} \frac{\mathrm{d} \bar{c}_{\mathrm{bH}}}{\mathrm{d} x}-P e_{z} \phi \bar{c}_{\mathrm{bH}}=0
$$

where

$$
\phi=\frac{\xi}{1+D(s)}+s+\frac{k^{2}}{P e_{\rho}}
$$

By using the elementary techniques of solving linear, homogeneous, second-order ordinary differential equations, the general solution of eq 29 comes out to be

$$
\bar{c}_{\mathrm{bH}}(s, k, x)=C_{3} \exp \left(m_{1} x\right)+C_{4} \exp \left(m_{2} x\right)
$$

for arbitrary constants $C_{3}$ and $C_{4}$. Here,

$$
\begin{aligned}
& m_{1}=\frac{P e_{z}}{2}\left(1+\sqrt{1+\frac{4 \phi}{P e_{z}}}\right) \\
& m_{2}=\frac{P e_{z}}{2}\left(1-\sqrt{1+\frac{4 \phi}{P e_{z}}}\right)
\end{aligned}
$$

To find the values of arbitrary constants $C_{3}$ and $C_{4}$, we can consider the Hankel transformations of either the Dirichlet or Danckwerts boundary conditions as explained below. 
Type I: Dirichlet Boundary Condition. Equations 12-14 are transformed to the following equations through Hankel transformation:

$$
c_{\mathrm{bH}}(\tau, k, x=0)= \begin{cases}c^{\mathrm{inj}} F(k), & 0 \leq \tau \leq \tau_{\mathrm{inj}} \\ 0, & \tau>\tau_{\mathrm{inj}}\end{cases}
$$

where $F(k)$ is the Hankel transform given by either

$$
F(k)= \begin{cases}\frac{\bar{\psi}^{2}}{2}, & k=0 \\ \frac{\bar{\psi} J_{1}(k \bar{\psi})}{k}, & k \neq 0\end{cases}
$$

or

$$
F(k)= \begin{cases}\frac{1-\bar{\psi}^{2}}{2}, & k=0 \\ -\frac{\bar{\psi} J_{1}(k \bar{\psi})}{k}, & k \neq 0\end{cases}
$$

Moreover, we get

$$
\left.\frac{\partial c_{\mathrm{bH}}}{\partial x}\right|_{x=\infty}=0
$$

Equation 34 can be used for inner-annular-region injection, eq 35 is for an injection through the outer annular region, and eq 36 shows the Neumann condition for hypothetically infinite length column.

Now, the Laplace transform of eqs 33 and 36 are given by

$$
\bar{c}_{\mathrm{bH}}(s, k, x=0)=\frac{c^{\mathrm{inj}} F(k)}{s}\left[1-\exp \left(-s \tau_{\text {inj }}\right)\right]
$$

and

$$
\left.\frac{\partial \bar{c}_{\mathrm{bH}}}{\partial x}\right|_{x=\infty}=0
$$

By applying eqs 37 and 38 in eq 31, the particular solution of the concentration in the bulk phase is given by

$$
\bar{c}_{\mathrm{bH}}(s, k, x)=\frac{c^{\mathrm{inj}} F(k) \exp \left(m_{2} x\right)}{s}\left[1-\exp \left(-s \tau_{\mathrm{inj}}\right)\right]
$$

Type II: Danckwerts Boundary Condition. Simultaneous application of the Laplace and Hankel transforms on eqs 15-17 gives us

$$
\bar{c}_{\mathrm{bH}}-\left.\frac{1}{P e_{z}} \frac{\mathrm{d} \bar{c}_{\mathrm{bH}}}{\mathrm{d} x}\right|_{x=0}=\frac{c^{\mathrm{inj}} F(k)}{s}\left[1-\exp \left(-s \tau_{\mathrm{inj}}\right)\right]
$$

and

$$
\left.\frac{\mathrm{d} \overline{\mathrm{c}}_{\mathrm{bH}}}{\mathrm{d} x}\right|_{x=1}=0
$$

where $F(k)$ is the same as that given in eqs 34 and 35. By applying eqs 40 and 41 in eq 31 , the particular solution of the concentration in the bulk phase comes out to be

$$
\begin{aligned}
\bar{c}_{\mathrm{bH}}(s, k, x)= & \left\{c ^ { \mathrm { inj } } F ( k ) \left[m_{2} \exp \left(m_{2}+m_{1} x\right)\right.\right. \\
& \left.\left.-m_{1} \exp \left(m_{1}+m_{2} x\right)\right]\left[1-\exp \left(-s \tau_{\mathrm{inj}}\right)\right]\right\} \\
& /\left\{s \left[m_{2} \exp \left(m_{2}\right)\left(1-m_{1} / P e_{z}\right)\right.\right. \\
& \left.\left.-m_{1} \exp \left(m_{1}\right)\left(1-m_{2} / P e_{z}\right)\right]\right\}
\end{aligned}
$$

For both types of the BCs, bringing back solutions in the actual $\tau$ and $\psi$ domains is very difficult because of the involvement of complicated functions. For that reason, we have successively used the numerical inversions of Laplace and Hankel transformations; see our recent published article for more details. $^{2}$

\section{MOMENT ANALYSIS}

Statistical temporal moments have substantial importance in extracting useful information about the mean retention time, the spreading, and the skewness of the concentration curve. At the exit of a column, the analytical expressions of moments are deduced by exploiting the property of moment generation of the Laplace and Hankel transforms: ${ }^{2,7,18}$

$$
\mu_{n, \mathrm{H}}=(-1)^{n} \lim _{s \rightarrow 0} \frac{\mathrm{d}^{n} \bar{c}_{\mathrm{bH}}}{\mathrm{d} s^{n}}, \quad n=0,1,2,3, \ldots
$$

By considering the dimensionless coordinate $\psi$ as an independent variable, the actual moments are obtained by using the following formula ${ }^{2}$

$$
\mu_{n}(\psi)=2 \mu_{n, \mathrm{H}}(k=0)+2 \sum_{n=1}^{\infty} \mu_{n, \mathrm{H}}(k) \frac{J_{0}(k \psi)}{\left|J_{0}(k)\right|^{2}}
$$

Using the actual moments given above, the non-normalized average moments, $M_{n, \text { av }}$, can be quantified using ${ }^{2}$

$$
M_{n, \mathrm{av}}=2 \int_{0}^{1} \mu_{n}(\psi) \psi \mathrm{d} \psi, \quad n=0,1,2,3, \ldots
$$

In chemical engineering, the normalized average temporal moments are frequently used and expressed $a^{2}$

$$
\begin{aligned}
& \mu_{n, \mathrm{av}}=\frac{M_{n, \mathrm{av}}}{\mu_{0, \mathrm{av}}} \\
& M_{0, \mathrm{av}}=\mu_{0, \mathrm{av}} \\
& n=1,2,3, \ldots
\end{aligned}
$$

From the above averaged moments, quantification of the first two central moments are given by the formula ${ }^{2,18}$

$$
\begin{aligned}
& \mu_{2, \mathrm{av}}^{\prime}=\mu_{2, \mathrm{av}}-\mu_{1, \mathrm{av}}{ }^{2} \\
& \mu_{3, \mathrm{av}}^{\prime}=\mu_{3, \mathrm{av}}-3 \mu_{1, \mathrm{av}} \mu_{2, \mathrm{av}}+2 \mu_{1, \mathrm{av}}{ }^{3}
\end{aligned}
$$

The zeroth temporal moment, the first temporal moment, the second central moment, and the third central moment respectively describe the total mass injected, the retention time, the spreading, and the asymmetry of the elution profile. In the case of nonlinear models, analytical expressions of temporal moments are not derivable. However, the following expressions can be used to calculate the moments numerically. At any position inside the column, the normalized $n^{\text {th }}$ moment averaged over the nondimensional radial coordinate $(\psi)$ is obtained numerically by using the formula ${ }^{2,18}$ 
Table 1. Standard Parameters Taken for the Numerical Test

\begin{tabular}{|c|c|c|c|c|c|c|c|c|c|c|c|c|c|}
\hline Figures & component & $P e_{z, i}$ & $B i_{\mathrm{p}, i}$ & $\eta_{\mathrm{p}, i}$ & $\epsilon_{\mathrm{b}}$ & $\epsilon_{\mathrm{p}}$ & $a_{i}$ & $K_{\mathrm{a}, i}$ & $C_{i}^{\mathrm{inj}}$ & $P e_{\rho, i}$ & $q_{\mathrm{m}}$ & $L[\mathrm{~cm}]$ & $u[\mathrm{~cm} / \mathrm{min}]$ \\
\hline $1-10$ & 1 & 600 & 50 & 2.0 & 0.4 & 0.33 & 1.2 & 1.0 & 1.0 & 15.0 & $(0.01,10,30)$ & 15 & 0.4 \\
\hline \multirow[t]{2}{*}{11 and 13} & 1 & 1500 & 50 & 2.0 & 0.4 & 0.33 & 0.5 & 1.0 & 1.0 & 37.5 & 20 & 10 & 1.0 \\
\hline & 2 & 1500 & 50 & 2.0 & 0.4 & 0.33 & 1.25 & 0.5 & 1.0 & 37.5 & 20 & 10 & 1.0 \\
\hline \multirow[t]{3}{*}{12} & 1 & 1500 & 50 & 2.0 & 0.4 & 0.5 & 1.0 & 0.5 & 1.0 & 37.5 & 20 & 10 & 1.0 \\
\hline & 2 & 1500 & 50 & 2.0 & 0.4 & 0.5 & 3.0 & 1.25 & 1.0 & 37.5 & 20 & 10 & 1.0 \\
\hline & 3 & 1500 & 50 & 2.0 & 0.4 & 0.5 & 5.0 & 1.5 & 1.0 & 37.5 & 20 & 10 & 1.0 \\
\hline
\end{tabular}
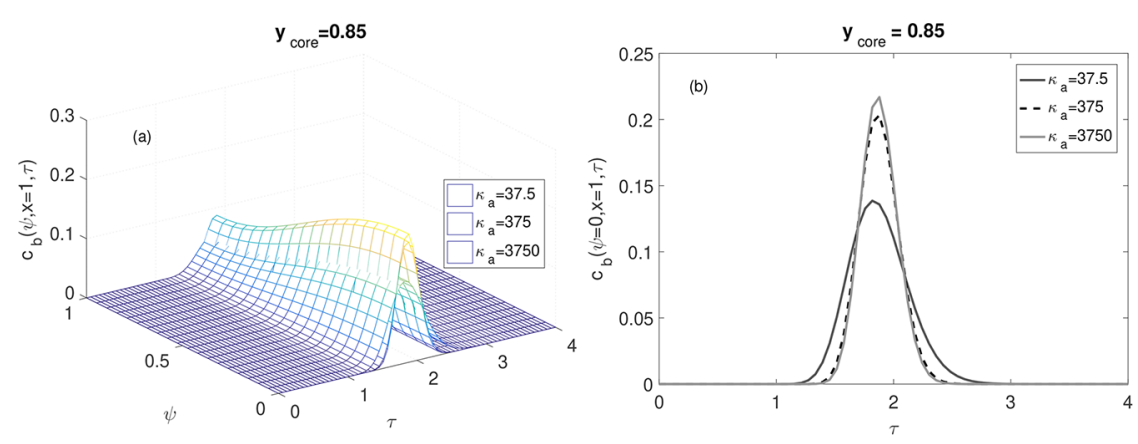

Figure 1. Inner-zone injection: effect of adsorption rate constant on the elution profile by considering Danckwerts BCs. Other parameters are kept fixed as given in Table 1 .

$$
\begin{aligned}
& \mu_{0, \mathrm{av}}=\int_{0}^{\infty} c_{\mathrm{b}, \mathrm{av}}(x, \tau) \mathrm{d} \tau \\
& \mu_{n, \mathrm{av}}=\frac{\int_{0}^{\infty} c_{\mathrm{b}, \mathrm{av}}(x, \tau) \tau^{n} \mathrm{~d} \tau}{\mu_{0, \mathrm{~b}, \mathrm{av}}} \\
& n=1,2,3, \ldots
\end{aligned}
$$

where

$$
c_{\mathrm{b}, \mathrm{av}}=2 \int_{0}^{1} c_{\mathrm{b}}(\psi, x, \tau) \psi \mathrm{d} \psi
$$

In this particular study, the zeroth, first, second, and third moments are obtained analytically for the Dirichlet boundary conditions, whereas only the zeroth, first, and second moments are obtained analytically for the Danckwerts boundary conditions. All these moments are listed in Appendix S1 of the Supporting Information.

\section{NUMERICAL SCHEME}

Several numerical techniques are accessible in the literature to numerically solve the chromatographic models. ${ }^{1,2,21-26}$ In this work, a flux-limiting, high-resolution, finite-volume technique is used to find numerical solutions of the current model equations. The details of the proposed numerical scheme are presented in Appendix S2 of the Supporting Information.

\section{CRITERION FOR ASSESSING PROCESS PERFORMANCE}

Process optimization is highly demanding in preparative chromatography. In this study, we propose a performance criterion for judging the quality of a product. For this purpose, we have assumed a mixture of two components in which the second component has higher affinity to the stationary phase than the first component. Let $\tau_{1}$ be the dimensionless time for which the concentration of the first component surpasses a certain level (i.e., $c_{\mathrm{b}, 1} \geq \epsilon c_{\text {inj, }}$ for $\epsilon=10^{-6}$ ). Similarly, let $\tau_{2}$ be a dimensionless time for which the concentration of the second component drops below a certain level (i.e., $c_{\mathrm{b}, 2} \leq \epsilon c_{\mathrm{inj}, 2}$ ). With the above assumptions, we have used purity, yield, productivity, and cycle time as assessment tools of process performance. The detailed formulas of the proposed assessment tools are already available in our recent research articles. ${ }^{1,2}$

\section{NUMERICAL TEST CASES}

This section provides some numerical test problems for demonstrating the effects of parameters on the elution process. The radius of the column and the radius of the packing particles are taken to be $R=0.2 \mathrm{~cm}$ and $R_{\mathrm{p}}=5 \times 10^{-6} \mathrm{~cm}$, respectively. To examine the effects of injection type, the annular ring is adjusted to be at $\rho=0.1414$ in the dimensional form or $\psi=0.7070$ in the dimensionless form. The column is assumed to be empty initially, and the injection time is taken as $t_{\mathrm{inj}}=1 \mathrm{~min}$. The values of the parameters presented in Table 1 are standard values for most of the presented problems. In all test problems, we have used $30 \times 20 \times 20$ grid points.

Single-Component Elution Using a Linear Model. This subsection is devoted to the case studies for the linear model. The derived analytical solutions are analyzed for different values of the coefficients of mass transfer and core-radius fraction, $y_{\text {core. }}$. The standard values of the parameters used in this subsection are presented in Table 1.

Figure 1 demonstrates the effect of the adsorption rate constant on the elution curve at the end of the column for a fixed core-radius fraction of $y_{\text {core }}=0.85$. It can be seen that the width of the curve decreases while the peak height increases when increasing the value of $\kappa_{\mathrm{a}}$. The retention time remains the same for all values of $\kappa_{\mathrm{a}}$. The limiting rate of the adsorption constant for the standard values of the parameters given in Table 1 is found to be $\kappa_{\mathrm{a}}=3750$. For this large value of $\kappa_{\mathrm{a}}$, the solution approaches the solution of the quasistationary sorption GRM presented in our previous article. ${ }^{2}$ It can also be observed that because of the inner-zone injection and slow radial dispersion, the concentration height in the inner zone is larger than that in the outer zone. 

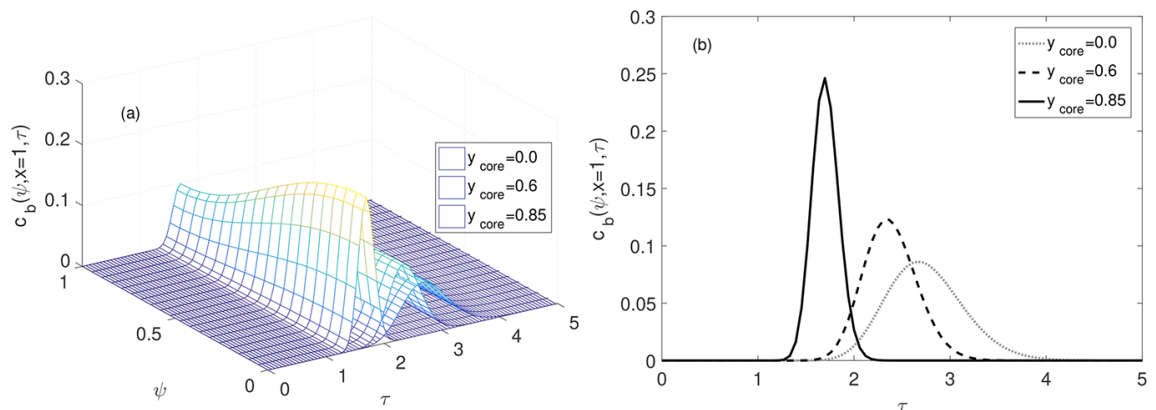

Figure 2. Inner-zone injection: effect of the core-radius on the elution profile for Danckwerts BCs. The adsorption rate is chosen as $\kappa_{\mathrm{a}}=375$. Other parameters are listed in Table 1.
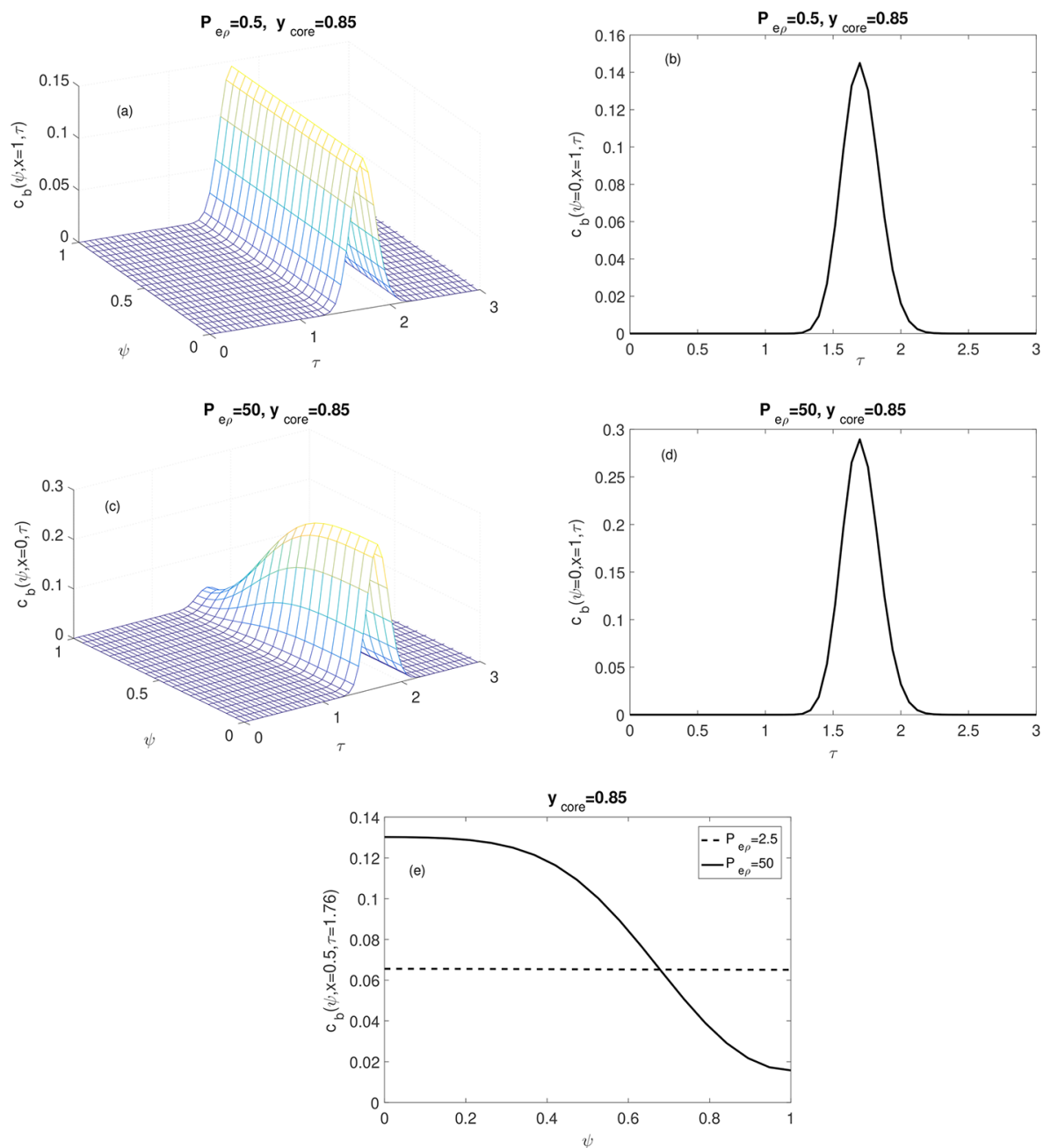

Figure 3. Inner-zone injection: effect of the radial Peclet number on the profiles. Here, $\kappa_{\mathrm{a}}=375$. (e) $1 \mathrm{D}$ plot given at the center of the column, $x=0.5$ for $\tau=1.76$. The remaining parameters are fixed as given in Table 1 .

Figure 2 depicts that how the core-radius fraction, $y_{\text {core }}$ affects the profile of concentration. Here, we have changed the values of the fractions of the core-radius from fully porous $\left(y_{\text {core }}=0.0\right)$ to core-shell particles $\left(y_{\text {core }}=0.85\right)$. Evidently, the efficiency of the column increases (i.e., retention time decreases) as the value of the core-radius fraction increases. Moreover, spreading of the profiles increases on decreasing the value of the core-radius fraction.

Figure 3 illustrates the effect of radial dispersion on the elution profile for a fixed value of core-radius faction $y_{\text {core }}=0.85$; Danckwerts boundary conditions with an inner-zone injection are considered. It is clear that small values of the radial dispersion correspond to higher peaks of the elution curve in the inner zone of the column as compared with in the outer zone (see Figures $3 \mathrm{~d}$ and $4 \mathrm{~d}$ ). On the other hand, larger values of the radial dispersion show no changes in the elution curve along the column radial coordinate (see Figures $3 a$ and $4 a$ ). For better observation, the $1 \mathrm{D}$ plot in Figure $3 \mathrm{e}$ presents the profile of a concentration at the middle of a column. It is visible that for large values of the radial Peclet number (i.e., for small values of the radial-dispersion coefficient), there is a change in the concentration along the radial coordinate, whereas the 

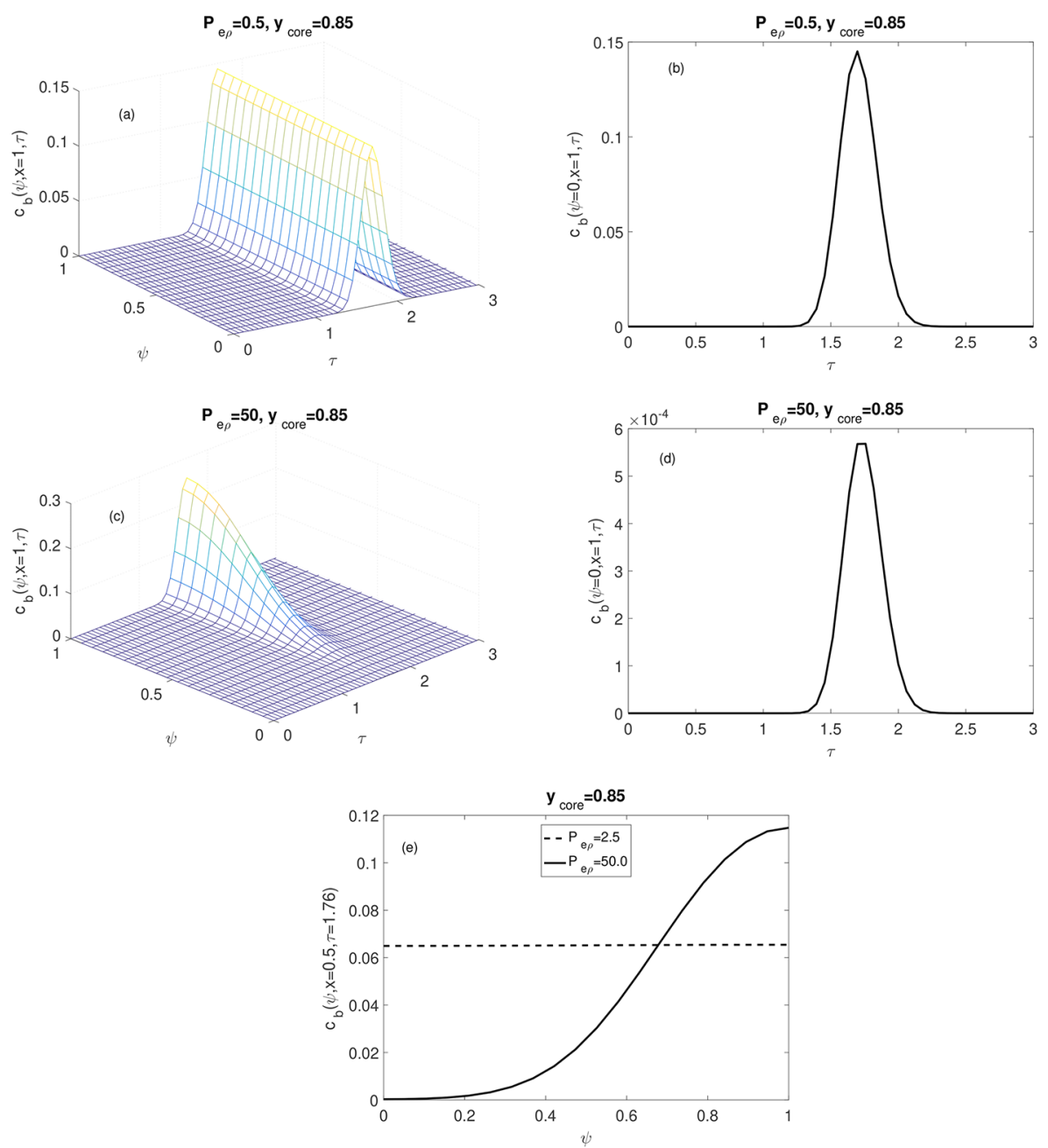

Figure 4. Outer-zone injection: effects of the radial Peclet number on the profiles. Here, $\kappa_{\mathrm{a}}=375$, and Danckwerts BCs are used. (e) 1D plot given at the center of the column, $x=0.5$ for $\tau=1.76$. The remaining parameters are given in Table 1 .
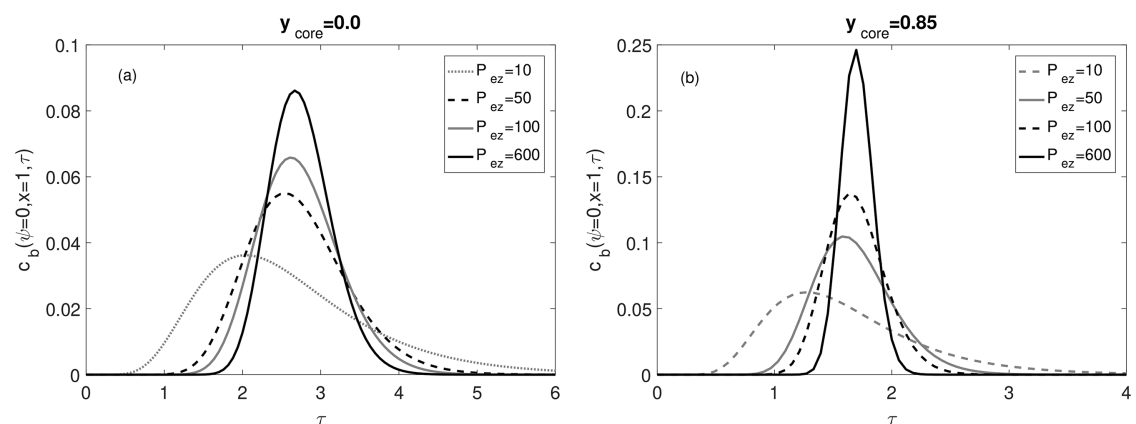

Figure 5. Inner-zone injection: effect of the axial Peclet number on the elution profiles. Danckwerts BCs and $\kappa_{\mathrm{a}}=375$ are used. All remaining parameters are listed in Table 1 .

concentration remains constant for small values of the radial Peclet number (i.e., for large values of the radial-dispersion coefficient). In Figure 4, the same case is presented when the injection in the outer annular zone is taken into account. Similar trends are observed like those seen in Figure 3.

In Figure 5, the effect of the axial Peclet number is presented for two distinct values of fractions of core-radii (i.e., $y_{\text {core }}=0.0$ and $y_{\text {core }}=0.85$ ). It can be seen that higher values of the Peclet number generate higher and narrower peaks of the profiles for both fractions of core-radii. In Figure 6, the impact of radial Peclet number on the analytical temporal moments is presented for a fixed core-radius fraction $\left(y_{\text {core }}=0.85\right)$. Larger values of the radial Peclet number produce radial variations in the moments, whereas smaller values of the radial Peclet number generate radially constant moments. In the same manner, the influence of $y_{\text {core }}$ on the analytical temporal moments is presented in Figure 7. It is evident that values of moments decrease with increasing values of $y_{\text {core. }}$.

Figure 8 displays the effect of the adsorption rate constant on the analytical temporal moments for a fixed $y_{\text {core }}=0.85$. It illustrates that an increase in the value of $\kappa_{\mathrm{a}}$ corresponds to a decrease in the values of the second and third moments. Besides 

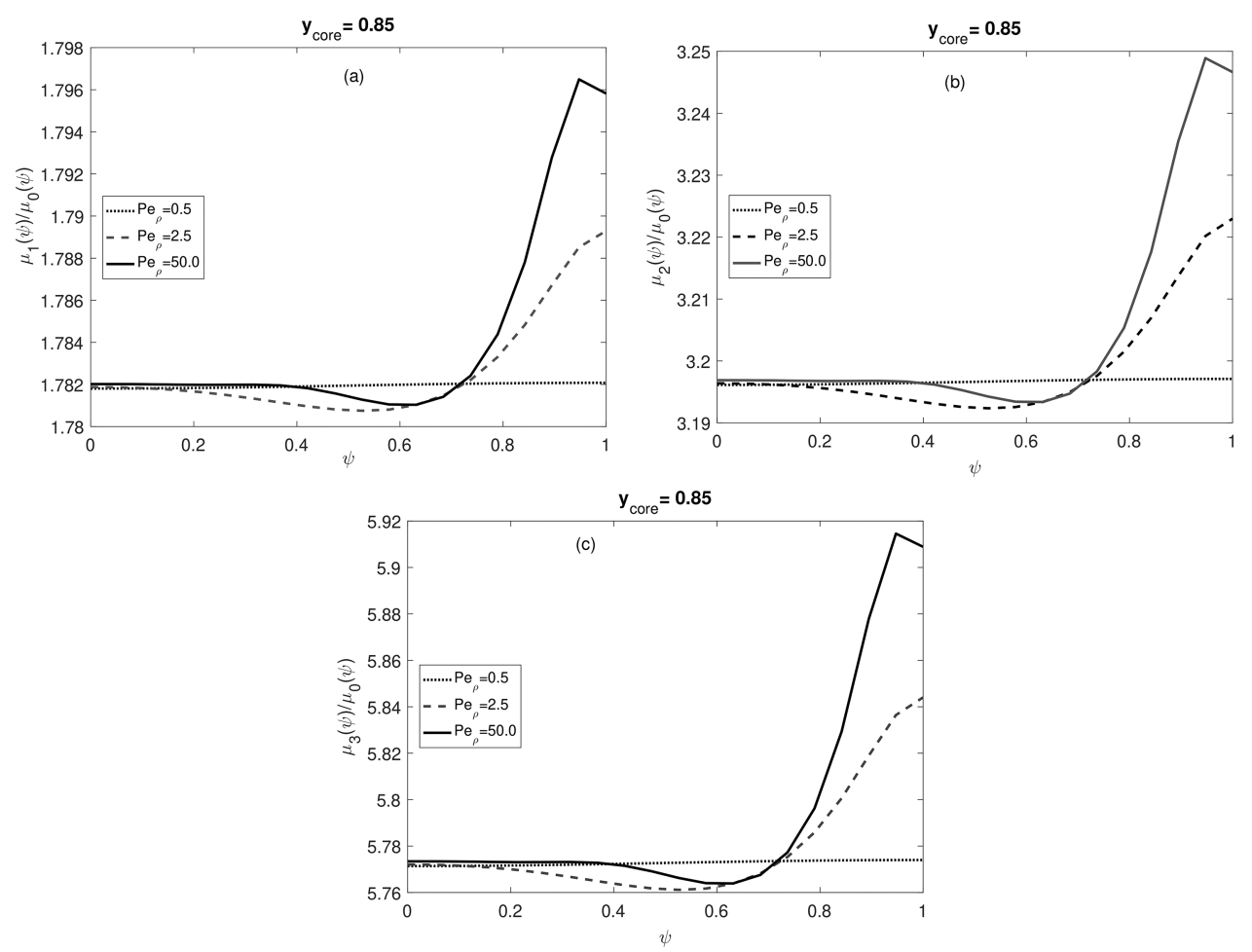

Figure 6. Effect of $P_{\mathrm{e} \rho}$ on the analytical moments. Dirichlet BCs and $\kappa_{\mathrm{a}}=375$ are used. All remaining parameters are listed in Table 1.
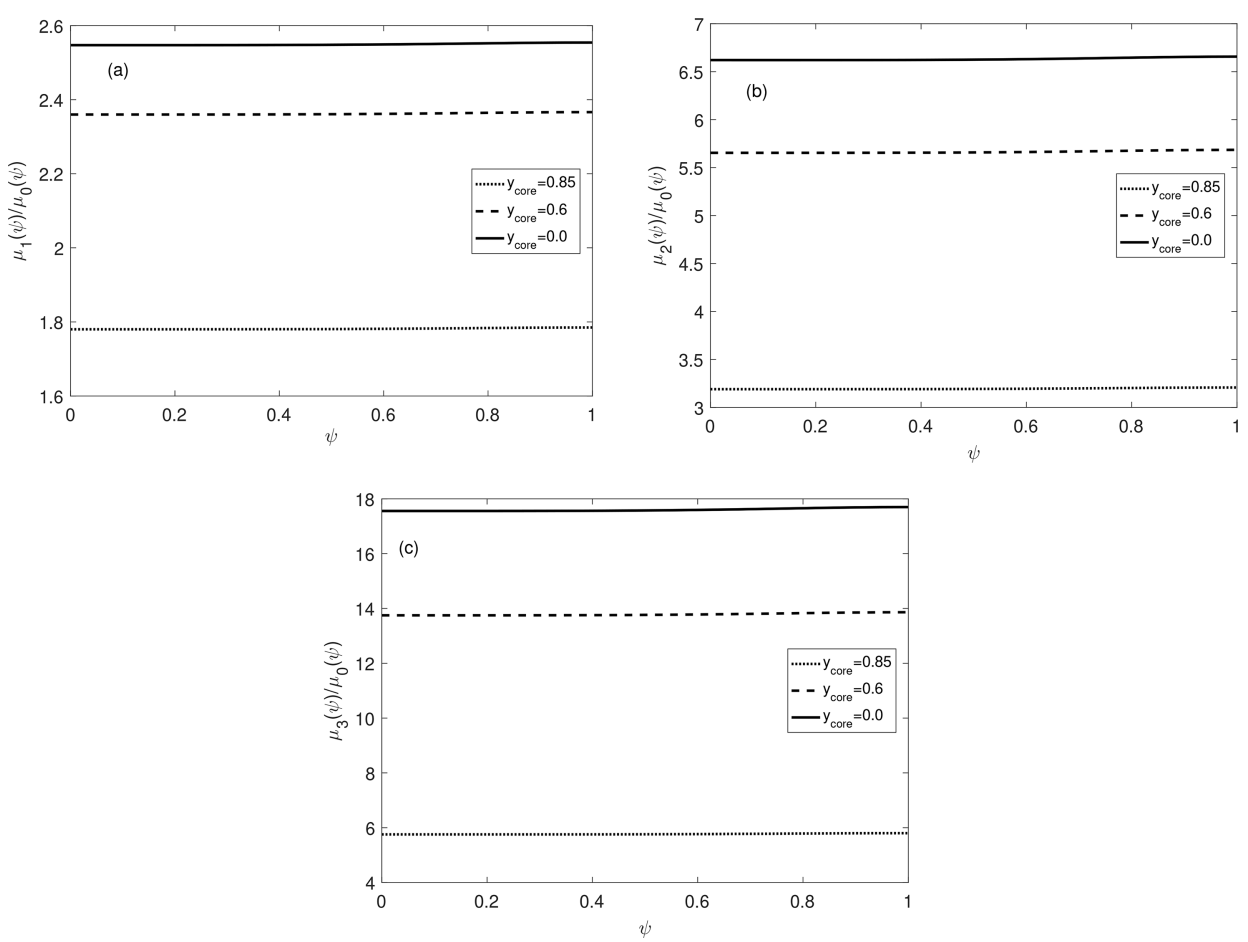

Figure 7. Effect of core-radius on the analytical moments. Dirichlet BCs and $\kappa_{\mathrm{a}}=375$ are used. All remaining parameters are listed in Table 1 .

that, the value of the first analytical temporal moment remained the same for all values of $\kappa_{\mathrm{a}}$. In Figure 9, the grid independence of the numerical method is demonstrated by comparing its solutions with the analytical solutions on two different grid points. The result show a good agreement between analytical and numerical solutions for both grid points, verifying that our chosen grid points are enough. The effects of the nonlinearity coefficients are examined in Figure 10 by taking very small and large values of saturation capacity and keeping the Henry's constant fixed. It depicts that the analytical and numerical solutions differ as the nonlinearity coefficient, $b_{i}=\frac{a_{i}}{q_{\mathrm{m}}}$, increases.

Thus, over-analytical solutions are only valid for small values of $b_{i}$ or for larger values of the adsorption-saturation capacity, $q_{\mathrm{m}}$.

Multicomponent Elution Using a Nonlinear Model. In this subsection, case studies of multicomponent, nonlinear 

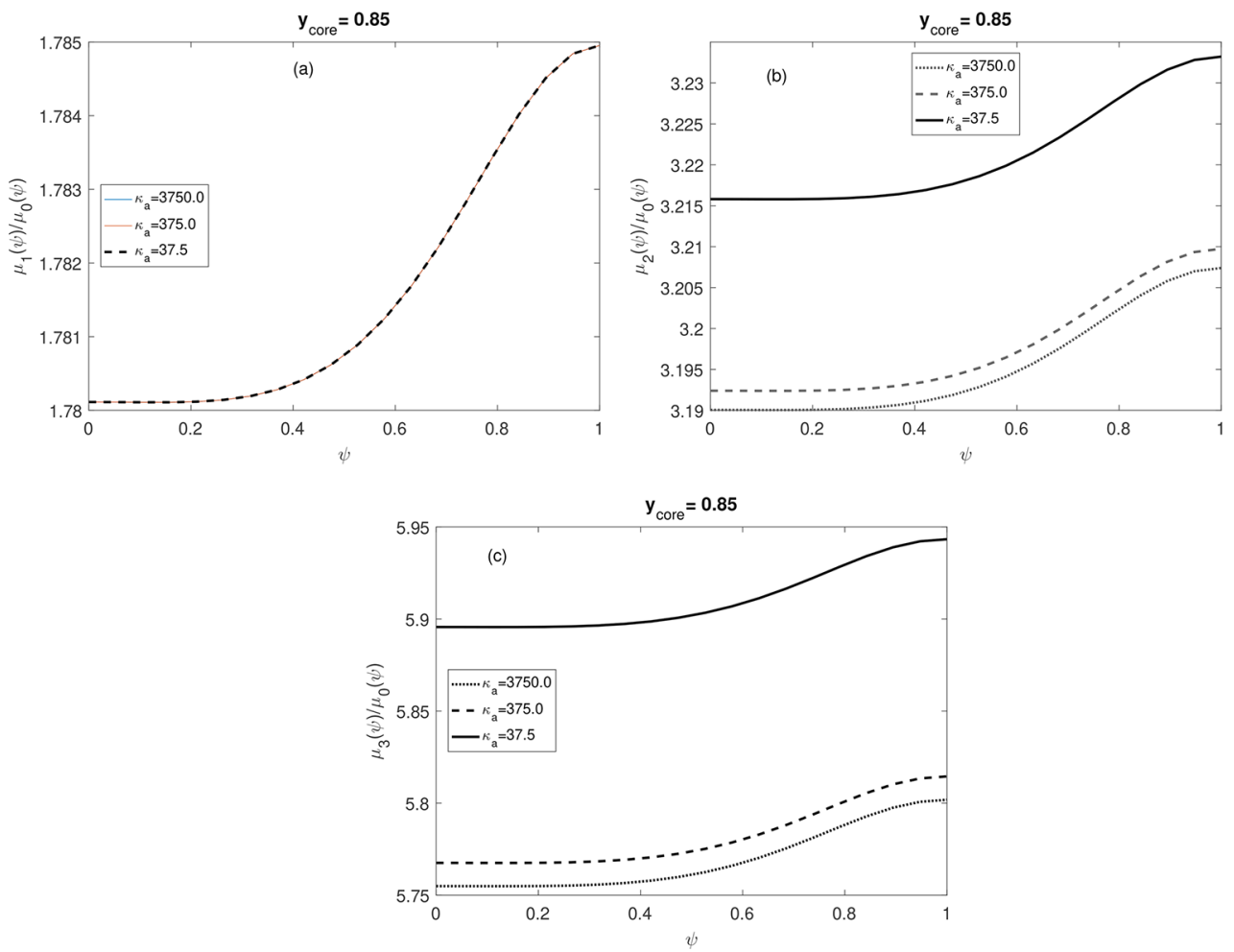

Figure 8. Effect of $\kappa_{\mathrm{a}}$ on the analytical moments. Dirichlet BCs are used. All remaining parameters are listed in Table 1.
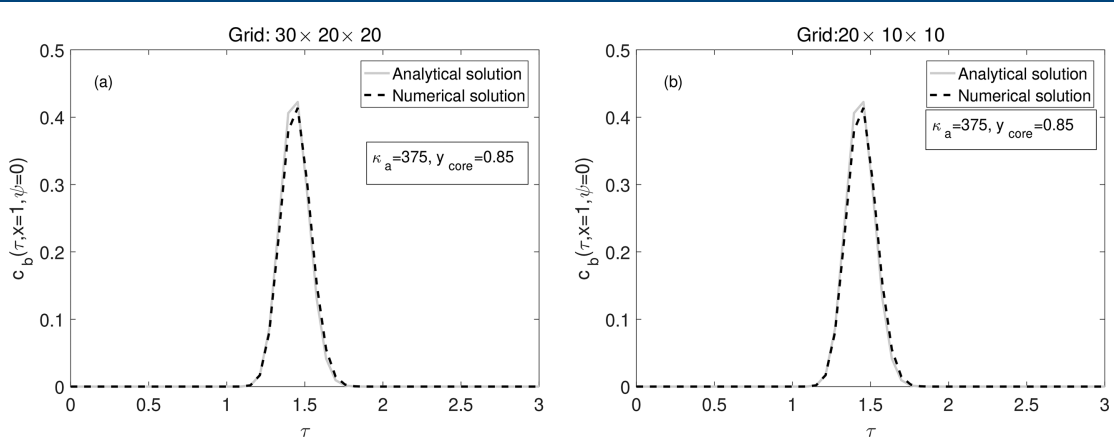

Figure 9. Comparison of analytical and numerical solutions based on grid points. Danckwerts BCs with inner-zone injection are used. All remaining parameters are listed in Table 1.
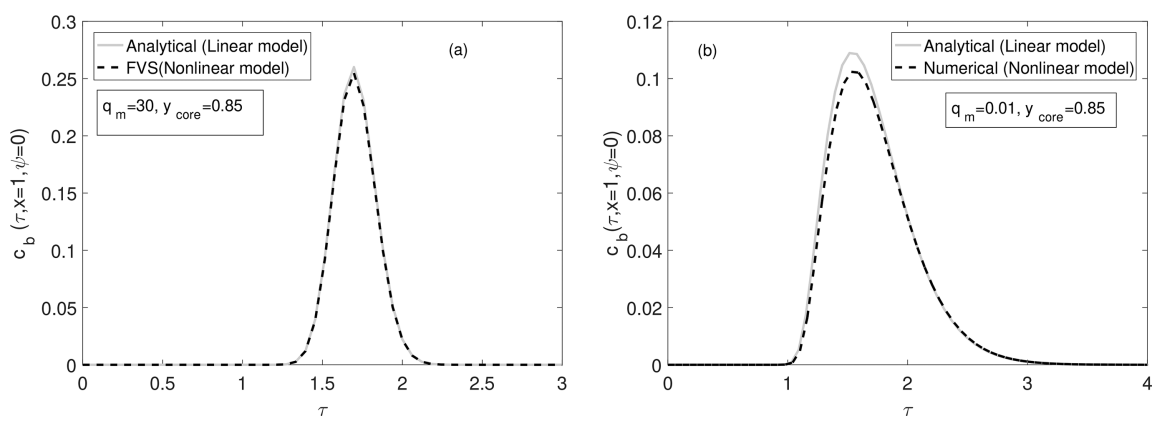

Figure 10. Plots showing the effect of the nonlinearity coefficient. Danckwerts BCs with inner-zone injection are used. All remaining parameters are listed in Table 1.

chromatography are presented. The effects of core-radius fraction, slow adsorption rate, and other kinetic parameters are studied on the elution curves of two- and three-component mixtures. The proposed process-performance criterion is also utilized for different mass-transfer parameters. All the computations are conducted on the basis of the standard parameters listed in Table 1.

Figure $11 \mathrm{a}-\mathrm{d}$ demonstrates the effects of different core-radii on the elution profiles of a mixture containing two components. It can be observed that separation of the components improves, retention times decrease, and profile spreading decreases as we 

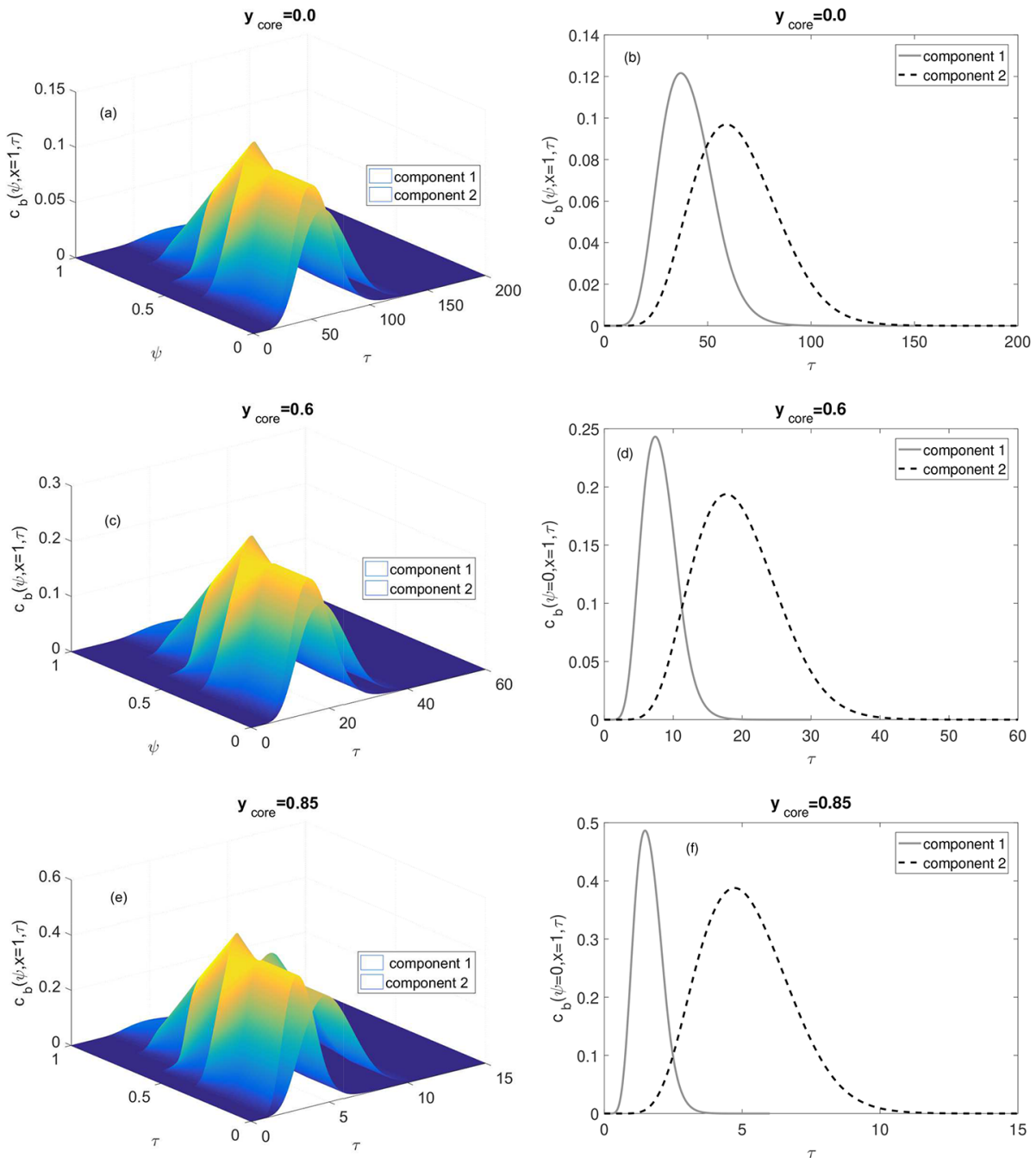

Figure 11. Two-component elution: the effect of the fraction of core-radius on the elution profiles. Danckwerts BCs are used. All remaining parameters are listed in Table 1.

increase the value of $y_{\text {core }}$. It is also evident that resolution of the components decreases with decreasing values of $y_{\text {core }}$. The aggregate elution time of the two components has shown a reduction from $\tau=150$ for $y_{\text {core }}=0.0$ to $\tau=15$ for $y_{\text {core }}=0.85$. This shows that the total time required for the two components to elute eventually decreases if we use core-shell particles with higher values of $y_{\text {core }}$.

Figure $12 \mathrm{a}-\mathrm{d}$ shows how the overlap, band-broadening, and retention times of the three-component-elution curves change with the application of different core-radii. It unveils that band broadening, retention times, and overlap of the elution curves decrease when the particle type is changed from fully porous to core-shell with higher core-radius fractions. It is also evident that resolution of the components increases with increasing values of the core-radius fraction. The aggregate elution time of all three components shows a reduction from $\tau=200$ for $y_{\text {core }}=$ 0.0 to $\tau=50$ for $y_{\text {core }}=0.85$. This confirms that the total time required for all components to elute out of the column eventually decreases when we use core-shell particles with higher fractions of core-radii.

In Figure $13 \mathrm{a}-\mathrm{d}$, the cut time, cycle time, productivity, and yield are plotted against the core-radius fraction. It demonstrates that cut and cycle times decrease from 34.2 to 7.4 and from 120 to 28.7 , respectively, as we move from fully porous to core-shell particles. The productivity keeps increasing until the core-radius fraction reaches $y_{\text {core }}=0.65$, after which it starts decreasing. The yield continues to increase as the value of $y_{\text {core }}$ increases.

\section{CONCLUSION}

A two-dimensional general rate model of column chromatography was solved analytically and numerically for slow rates of adsorption-desorption kinetics, radial and axial dispersions, and core-shell particles. Two different sets of boundary conditions, Danckwerts and Dirichlet boundary conditions, were taken into account. Solutions of the model were obtained analytically for a linear isotherm by successively implementing the Hankel and Laplace transformations. Because of the involvement of complicated functions in the solutions, numerical Laplace and Hankel inversion techniques were utilized for transforming back solutions in the actual time and radial coordinates. Using these analytical results, several case studies were conducted to examine the effects of slow rates of adsorption-desorption, radial and axial dispersions, and core-radius fractions on the elution curves. In the case of the nonlinear isotherm, the effects of the model parameters were examined by using the numerical results of a semidiscrete, high-resolution, finite-volume scheme. The simulation results reveal that an increment in the value of the core-radius fraction provides shorter residence times and 

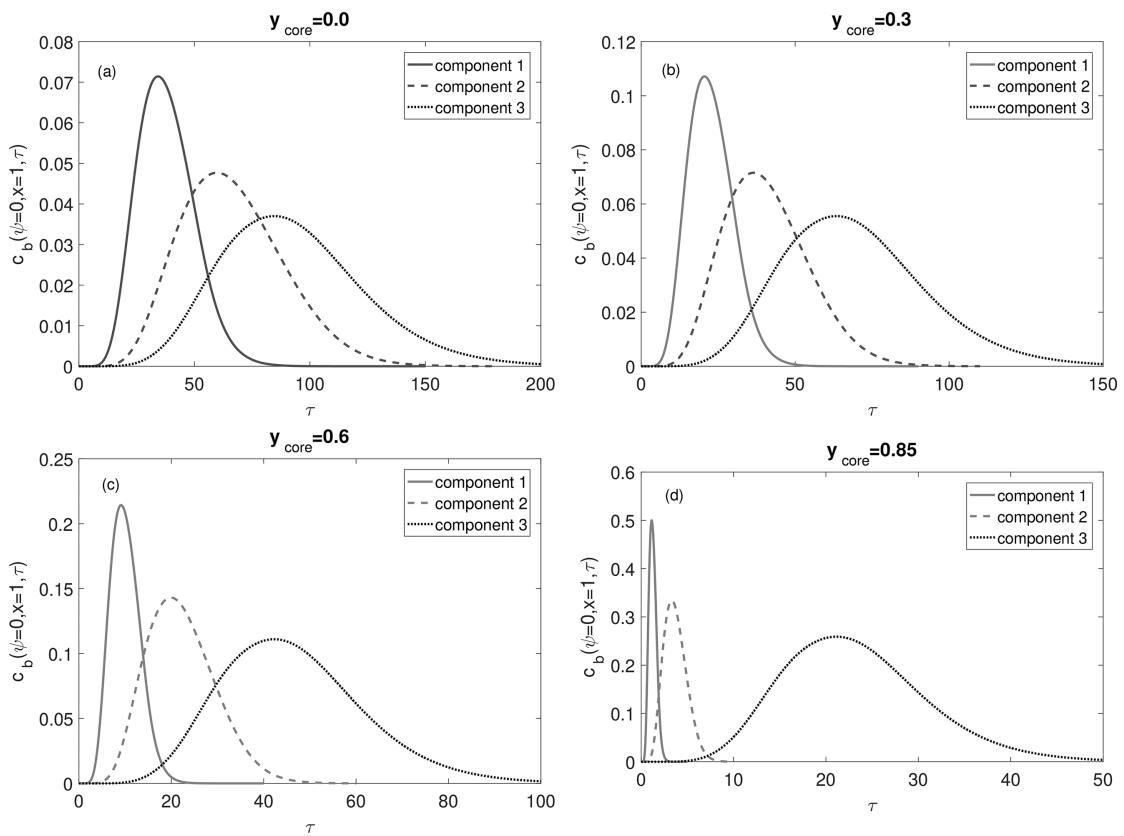

Figure 12. Three-component elution: the effect of the core-radius on the elution profiles. Danckwerts BCs are used. All remaining parameters are listed in Table 1.
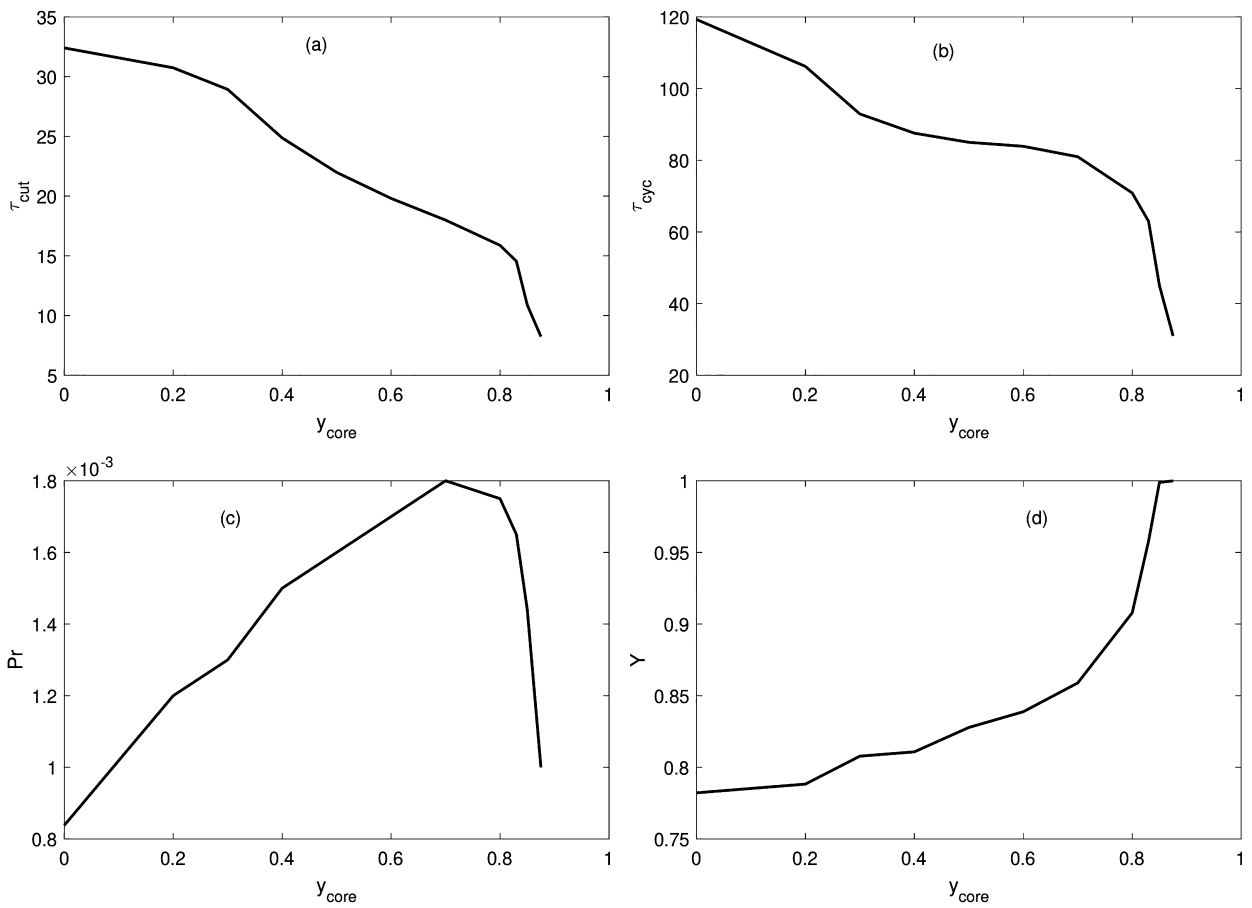

Figure 13. Plots of (a) $\tau_{\text {cut }}$ (b) $\tau_{\text {cyc }}$ (c) productivity (Pr), and (d) yield $(Y)$ as functions of the core-radius. Danckwerts BCs are used to produce the results. All remaining parameters are listed in Table 1.

sharper and narrower elution peaks. The column performance was also analyzed by utilizing a specified performance criterion. The current results will be very helpful for upgrading those chromatographic columns in which radial concentration gradients are significant.

\section{ASSOCIATED CONTENT}

\section{S Supporting Information}

The Supporting Information is available free of charge on the ACS Publications website at DOI: 10.1021/acs.iecr.9b00364.
Analytical moments and the numerical scheme (PDF)

\section{AUTHOR INFORMATION}

\section{Corresponding Author}

*E-mail: shamsul.qamar@comsats.edu.pk.

ORCID

Shamsul Qamar: 0000-0002-7358-6669

Notes

The authors declare no competing financial interest. 


\section{ACKNOWLEDGMENTS}

The first author is grateful to The World Academy of Sciences (TWAS) and COMSATS University Islamabad for the Award of a 2016 CIIT-TWAS Full-time Postgraduate Fellowship (FR number: 3240293226).

\section{REFERENCES}

(1) Qamar, S.; Sattar, F. A.; Abbasi, J. N.; Seidel-Morgenstern, A. Numerical simulation of nonlinear chromatography with core-shell particles applying the general rate model. Chem. Eng. Sci. 2016, 147, 54.

(2) David, U. U.; Qamar, S.; Seidel-Morgenstern, A. Analytical and numerical solutions of two-dimensional general rate models for liquid chromatographic columns packed with core-shell particles. Chem. Eng. Res. Des. 2018, 130, 295.

(3) Guiochon, G.; Felinger, A.; Shirazi, D. G.; Katti, A. M. Fundamentals of Preparative and Nonlinear Chromatography, 2nd ed.; Elsevier Academic Press: New York, 2006.

(4) Guiochon, G.; Lin, B. Modeling for Preparative Chromatography; Academic Press: London, 2003.

(5) Guiochon, G. Preparative Liquid Chromatography. J. Chromatogr. A 2002, 965, 129.

(6) Kirkland, J. J. Modern practice of liquid chromatography; WileyInterscience: New York, 1971.

(7) Guiochon, G.; Golshan-Shirazi, S.; Katti, A. M. Fundamentals of nonlinear and preparative chromatography; Academic Press: Boston, 1994.

(8) Miyabe, K. Moment Analysis of Chromatographic Behavior in Reversed-phase Liquid Chromatography. J. Sep. Sci. 2009, 32, 757.

(9) Olah, E.; Fekete, S.; Fekete, J.; Ganzler, K. Comparative study of new shell-type, sub- $\mu m$ fully porous and monolith stationary phases, focusing on mass-transfer resistance. J. Chromatogr. A 2010, 1217, 3642.

(10) Ruthven, D. M. Principles of Adsorption and Adsorption Processes; John Wiley and Sons: New York, 1984.

(11) Carta, G. Exact analytic solution of a mathematical model for chromatographic operations. Chem. Eng. Sci. 1988, 43, 2877.

(12) Lin, B.; Song, F.; Guiochon, G. Analytical solution of the ideal, nonlinear model of reaction chromatography for a reaction $A \rightarrow B$ and a parabolic isotherm. J. Chromatogr. A 2003, 1003, 91.

(13) Cavazzini, A.; Gritti, F.; Kaczmarski, K.; Marchetti, N.; Guiochon, G. Mass-transfer kinetics in a shell packing material for chromatography. Anal. Chem. 2007, 79, 5972.

(14) Kaczmarski, K.; Guiochon, G. Modeling of the mass-transfer kinetics in chromatographic columns packed with shell and pellicular particles. Anal. Chem. 2007, 79, 4648.

(15) Gritti, F.; Cavazzini, A.; Marchetti, N.; Guiochon, G. Comparison between the efficiencies of columns packed with fully and partially porous C18-bonded silica materials. J. Chromatogr. A 2007, 1157, 289.

(16) Gritti, F.; Guiochon, G. Comparative study of the performance of columns packed with several new fine silica particles: Would the external roughness of the particles affect column properties? J. Chromatogr. A 2007, 1166, 30.

(17) Qamar, S.; Seidel-Morgenstern, A. Extending the Potential of Moment Analysis in Chromatography. TrAC, Trends Anal. Chem. 2016, $81,87$.

(18) Qamar, S.; Akram, N.; Seidel-Morgenstern, A. Analysis of general rate model of linear chromatography considering finite rates of the adsorption and desorption steps. Chem. Eng. Res. Des. 2016, 111, 13.

(19) Li, P.; Yu, J.; Xiu, G.; Rodrigues, A. E. A strategy for tailored design of efficient and low-pressure drop packed column chromatography. AIChE J. 2010, 56, 3091.

(20) Qamar, S.; Bashir, S.; Perveen, S.; Seidel-Morgenstern, A. Analysis of linear general rate model of reactive chromatography for core-shell adsorbents. Ind. Eng. Chem. Res. 2017, 56, 9657.

(21) Javeed, S.; Qamar, S.; Ashraf, W.; Warnecke, G.; SeidelMorgenstern, A. Analysis and Numerical Investigation of Two Dynamic Models for Liquid Chromatography. Chem. Eng. Sci. 2013, 90, 17.
(22) von Lieres, E.; Andersson, J. A fast and accurate solver for the general rate model of column liquid chromatography. Comput. Chem. Eng. 2010, 34, 1180.

(23) Javeed, S.; Qamar, S.; Seidel-Morgenstern, A.; Warnecke, G. Efficient and Accurate Numerical Simulation of Nonlinear Chromatographic Processes. Comput. Chem. Eng. 2011, 35, 2294.

(24) Cockburn, B.; Shu, C. W. TVB Runge-Kutta local projection discontinuous Galerkin finite element method for conservation laws. II. General framework. Mathematics of computation 1989, 52, 411.

(25) Qamar, S.; Perveen, S.; Seidel-Morgenstern, A. Numerical approximation of nonlinear and non-equilibrium two-dimensional model of chromatography. Comput. Chem. Eng. 2016, 94, 411.

(26) Akram, N.; Qamar, S.; Seidel-Morgenstern, A. (2018). Nonlinear model of liquid chromatography considering finite rates of adsorptiondesorption kinetics and core-shell adsorbents. J. Liq. Chromatogr. Relat. Technol. 2018, 41, 964. 